\title{
Proyecto Amig@S Activ@S
}

\author{
Ángel Navarro \\ CEIP Ma Moliner, España \\ Dani Martín \\ CEIP Valdespartera, Espana
}

\section{Resumen}

El proyecto Amig@s Activ@s fue creado en junio de 2015 por un grupo de profesores de educación física de la Asociación + Aragón de Educación Física, con el objetivo de promover una vida saludable, prevenir la obesidad y promover los derechos humanos, inclusión e igualdad de género, cooperación para el desarrollo, educación ambiental, comunicación y educación emocional. Con este fin, se propuso un proyecto de aprendizaje-servicio con participación de la comunidad: maestros de primaria de todas las áreas, estudiantes, familias activas, otros agentes (asociaciones, instituciones, universidades, etc.) y la Fundación Familias Unidas.

\section{Palabras clave}

Aprendizaje-servicio, educación física, comunidad. 


\title{
The Active Friends Project
}

\begin{abstract}
The Active Friends project (Proyecto Amig@s Activ@s in Spanish) was created in June 2015 by a group of PE teachers from the + EF Aragon Association, with the aims of promoting healthy living, prevent obesity, as well as promoting human rights, inclusion and gender equality, cooperation for development, environmental education, communication and emotional education. To this end, a service learning project with community participation was proposed, involving primary teachers from all areas, students, active families, other agents (associations, institutions, universities, etc.) and the Familias Unidas Foundation.
\end{abstract}

\section{Keywords}

Service-learning, physical education, community. 


\section{Marco del Proyecto Amig@s Activ@s}

Nos ubicamos en la comunidad autónoma de Aragón. Hernández (2009), Murillo (2013) y Frago (2015) indican que hay una necesidad de incentivar la práctica de actividad física en la infancia y adolescencia aragonesa (también en la española según GarcíaFerrando y Llopis, 2017), ya que la mayoría no alcanza las recomendaciones internacionales de practicar 60 minutos diarios de actividad física moderada o vigorosa (OMS, 2010). Para ello, el rol del docente de Educación Física es esencial como referente y dinamizador de la implicación de la comunidad (Solmon, 2015) y del centro educativo como lugar de promoción de la salud (Carta de Ottawa, GAPA, 2010).

Además, han quedado demostrados múltiples beneficios de practicar actividad física, destacando la mejora de la salud (Devís y Peiró, 1993), la educación integral del alumnado (Dotterweich et al, 2012), actividad cerebral (Ramírez et al, 2004) y bienestar psicológico (Ojiambo, 2013)

El Proyecto Amig@s Activ@s (Navarro, Martín, García, Jofre, Hernández y Gómez, 2017) pretende ser una propuesta educativa global que aborde todos los ámbitos de la personalidad, la participación activa y el servicio a la comunidad, dinamizando los activos de salud de la misma (Cofiño et al, 2016). Se fundamenta en el modelo social ecológico de promoción de la salud (Sallis, Owen y Fisher, 2008), alineando

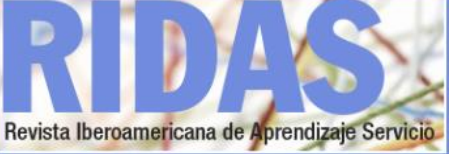

las cuatro esferas educativas esenciales: docente, institución educativa, comunidad y, a ser posible, política educativa (Langille y Rodgers, 2010). A nivel metodológico se asienta en el aprendizaje basado en proyectos y concretamente en el ApS (Puig, Batlle, Bosch, Palos, 2007; Aramburuzabala, 2015).

Entendemos que "los niños y jóvenes no son los ciudadanos del futuro, son ya ciudadanos capaces de provocar cambios en su entorno, (...) posibilitando que actúen como ciudadanos comprometidos" (Batlle, 2011, p. 50), y por ello, queremos convertirlos en protagonistas de mejoras sociales en su comunidad, que motiven, enlacen y afiancen los aprendizajes programados, con el servicio y un trasfondo solidario.

Como desarrollaremos posteriormente, la magnitud actual del Proyecto Amig@s Activ@s es de 8 sectores, 47 centros y más de 2500 alumnos. En cada sector tiene lugar, adaptándolo a sus necesidades, con 8 tareas preevento, el evento principal y 4 tareas post-evento. De forma flexible e inclusiva, llevamos a cabo los ajustes necesarios para garantizar la presencia, participación y éxito de todos (UNESCO, 2015).

Todos los sectores desarrollan las tareas pre, durante y post, incluyendo la convivencia deportiva (evento central), practicando actividades de cooperación y colaboración en $2{ }^{\circ}$ curso, juegos y deportes alternativos en $4^{\circ}$ curso y actividad física en medio natural en $6^{\circ}$ curso, así como el espacio emocional correspondiente a $2^{\circ}$ curso 
(medioambiental), 40 curso (deporte y derechos humanos) y $6^{\circ}$ curso ( 6 erres: repensar, reestructurar, redistribuir, reducir, reciclar, reutilizar). Hay participación activa de las familias tanto en las tareas competenciales como en la convivencia. Todo el trabajo, experiencias y vivencias es plasmado en el blog del proyecto

http://www. proyectoamigosactivos.blog spot.com, incluyendo notas de prensa y radio. Todo ello se difunde y comparte en la cuenta Twitter del proyecto @EF_activos.

La participación de alumnado, profesorado de todas las áreas y familias activas es común a los 8 sectores (ver Figura 1). En cada uno de ellos intentamos aprovechar los recursos que presentan los agentes de cada comunidad (Martín et al, 2018), destacando la Fundación Familias Unidas, ONG de Cooperación para el Desarrollo, vinculada a nuestro proyecto mediante su programa de Educación para la Ciudadanía Global Sensibilización con Dos Orillas.

Una característica diferenciadora del trabajo con la Fundación Familias Unidas es haber ejecutado la propuesta educativa de Amig@s Activ@s en las escuelas adscritas al proyecto de deporte y educación que ejecuta dicha ONGD en Estelí, Nicaragua.

\section{Sinopsis del Proyecto Amig@s Activ@: forma parte y iactívate!}

El Proyecto Amig@s Activ@s nace en junio de 2015 a raíz de un grupo de docentes de la asociación +EF Aragón. Principalmente, queremos aprender en forma de proyecto, hacer más amigos y que sean más sanos y activos, incluyendo los determinantes de salud: actividad física, nutrición, higiene, sueño, relaciones, emociones y participación social.

Promocionamos la salud, previniendo la obesidad, junto con los derechos humanos, inclusión e igualdad de género, cooperación para el desarrollo, educación ambiental, comunicación y educación emocional. Es esencial la participación comunitaria: docentes de primaria de todas las áreas, alumnado, familias activas, otros agentes (asociaciones, instituciones, universidad) y la Fundación Familias Unidas, sumando Dos orillas activas y uniendo el Proyecto Amig@s Activ@s a la Educación para el Desarrollo y la Ciudadanía Global, solidaridad y cooperación con Nicaragua.

En cuatro fases hemos ampliado el proyecto: 8 sectores, 47 centros y más de 2500 alumnos/as. En https://youtu.be/mrQ4xfr knc puede verse un vídeo con la experiencia.

\section{Necesidad social que atiende el proyecto}

Además de su finalidad principal como promoción de hábitos saludables y prevención de obesidad, el proyecto aborda otras necesidades sociales: derechos humanos, inclusión y equidad de género, cooperación al desarrollo, educación ambiental, comunicación y educación emocional.

Al respecto, Murillo et al (2013) indican que en la promoción de la salud se debe prestar especial atención a las chicas, personas con sobrepeso y en 
riesgo de exclusión social. Además, Solmon (2015) destaca que los hábitos adquiridos en la infancia son más fáciles de ser continuados en la edad adulta, y que las propuestas que atiendan a mecanismos motivacionales (González-Cutre, 2017; Julián, 2012) y a la motivación intrínseca, autonomía, competencia y relación, logran más práctica futura de actividad física.

En la misma línea, existe una necesidad social de educar de forma activa, fomentando la participación, protagonismo y toma de decisiones del alumnado, conectando aprendizaje y servicio a la comunidad. Este enfoque abarca todas las áreas, destacando comunicación, socialización e intercambio emocional como señas de identidad del proyecto, en un marco de participación comunitaria, que potencia más aprendizajes (Hattie y Anderman, 2013).

Como telón de fondo, si queremos sociedades más democráticas y humanas, es esencial participar y tomar decisiones desde la infancia: trabajar en equipo, lograr las finalidades del proyecto; buscar alternativas, proponer soluciones, evaluarlas, satisfacer necesidades sociales del barrio, localidad o comarca; avanzar en las habilidades sociales y emocionales que sustentan los intercambios humanos, etc. Buscamos satisfacer todas ellas, con la motricidad y la promoción de la salud como hilo conductor, y de forma activa.

Esta voluntad adquiere una dimensión y calado mayor al vincular la Educación para el Desarrollo y la Ciudadanía Global (EpDG) y la solidaridad y cooperación con Nicaragua, creando una ruta de intercambio de saberes educativos y solidaridad entre educadores, niños de Zaragoza y Estelí (Nicaragua), dando relevancia, visibilizando y potenciando la comprensión y el compromiso global de los Derechos Humanos y la igualdad de género. Igualmente, consideramos que el propio proyecto puede prestar un servicio a la comunidad educativa en general, y por ello, nace con la voluntad de ser compartido.

\section{Objetivos educativos}

Los objetivos son los siguientes:

1. Promocionar la salud a través de aprendizajes funcionales, motivantes y aplicables en su contexto de referencia, abordando dimensiones de la personalidad y determinantes de salud, enfatizando las metodologías activas y el servicio a la comunidad.

2. Fomentar la alfabetización motriz, gestión de vida activa, comunicación, educación emocional y en valores, educación medioambiental y derechos humanos, mejorando la convivencia desde una perspectiva inclusiva y de participación comunitaria.

3. Mejorar la socialización y convivencia, promoviendo un vínculo ligado a la actividad física, la salud y la diversión, extensible al tiempo de ocio y a la etapa de secundaria.

4. Potenciar el rol activo del alumnado, su toma de decisiones y su participación social como un agente 
más con influencia positiva en su entorno.

\section{Hacemos e investigamos: actividades de aprendizaje y de reflexión}

Las actividades de aprendizaje que proponemos están explicitadas en Martín et al (2018). Al respecto, queremos que sean "oportunidades de participación, cooperación, reflexión y reconocimiento" (De la Cerda en Puig et al, 2015: 47). En ellas toman forma y se plasman nuestras intenciones de servicio, ensalzando el rol protagonista y activo del alumnado, buscando su vinculación emocional a las tareas, potenciando la comunicación y relación con otros, que tengan una aplicación clara, y compartirlo con la comunidad. Cuando realizamos en $4^{\circ}$ curso la tarea de aprendizaje servicio vinculada al consumo de azúcar en los almuerzos del colegio, el proceso llevado a cabo por el alumnado ha sido una investigación (recogida, tratamiento de información y extracción de conclusiones) y una reflexión posterior previa a la transformación, para dotar de sentido la acción educativa buscando aprendizajes útiles para la vida.

El reconocimiento al compartir y contar a otros también está muy presente. Además del refuerzo positivo del profesorado, por la reciprocidad con los beneficiarios, constatando la utilidad y el sentido del proyecto, adquiriendo a través del blog una dimensión pública.

\section{Compartimos y difundimos}

Cada uno de los sectores comparte un post contando su convivencia, y las tareas pre y post evento. El denominador común en las fotos, vídeos y textos de los sectores es aprendizaje, satisfacción, diversión, éxito, socialización, emociones y creatividad.

El gran momento de celebrar y dar visibilidad es el Encuentro Solidario, organizado conjuntamente con Familias Unidas. Es un día muy especial, y tiene tres elementos fundamentales:

1. Exposición interactiva: conocer todos los aprendizajes del Proyecto Amig@s Activ@s, así como la labor de la Fundación en Nicaragua a través de Dos orillas activas.

2. Mercadillo solidario: adquirir la camiseta solidaria de Amig@s Activ@s por $5 €$, y aprovechar para conseguir productos de comercio justo; se vendieron más de 600 .

3. Encuentro deportivo: practicar los juegos y deportes del Proyecto Amig@s Activ@s con la familia y pasar una tarde llena de diversión (aportación por familia, al menos $1 €)$.

\section{Evaluamos: queremos seguir mejorando}

La evaluación es un instrumento esencial para recoger información válida, valiosa y fiable (Pérez Juste, 2006) orientada a la mejora del proyecto y de sus participantes (Stufflebeam y Shinkfield, 1989). Precisamente, de todas las funciones evaluativas, queremos potenciar la mejora del alumnado, el profesorado, la comunidad y del propio Proyecto 
Amig@s Activ@s.

Tanto en su dimensión pedagógica como en el servicio, el proyecto ha alcanzado sus objetivos, y en su difusión, ha superado nuestras altas expectativas.

Los resultados en relación a participación y satisfacción son elevados. Contribuimos a alcanzar las recomendaciones de tiempo de práctica de actividad física, fomentando más oportunidades de práctica.

Cualitativamente, facilitamos la gestión de la vida activa del alumnado, promoviendo buenos hábitos y ligando la percepción del éxito a la diversión, la autonomía, la competencia, la relación con los demás y la novedad (GonzálezCutre, 2017).

La participación del alumnado ha sido del $100 \%$ de los cursos y sectores implicados en las tareas de aprendizaje. En la convivencia hemos potenciado el acceso de todos buscando sinergias y colaboraciones comunitarias para abaratar al máximo el transporte y la ambulancia obligatoria, asumidos por centros, asociaciones de familias, colaboradores o instituciones.

Realizamos una evaluación multifocal (Puig, 2015) valorando el logro, comparando y analizando distintas fuentes de información, siempre con la participación de la comunidad: alumnado, familias, profesorado, otros agentes. Con ella extraemos conclusiones orientadas a la mejora. Las herramientas de recogida de información han sido cuestionario, libro de firmas y libro viajero, muro colaborativo (padlet), actividades de contar a otros y diario de aprendizaje (Martín et al, 2018; Navarro et al, 2017)

Para finalitzar, hemos evaluando siguiendo a Puig et al (2014) y Campo (2015), encontrando que nuestras vivencias y lo que hemos compartido en las reuniones de docentes y con los distintos agentes de nuestra comunidad son valoraciones altamente positivas en aprendizaje, servicio, participación y reflexión, así como en coordinación y trabajo conjunto, si bien observamos la necesidad de seguir informando y compartiendo el proyecto a la comunidad para potenciarlo más aún.

\section{Conclusiones}

Destacamos que el nivel de satisfacción de alumnado, profesorado y familias es alto o muy alto. La valoración que se realiza del proyecto es muy positiva, y el interés mostrado por otros docentes en el proyecto es alto, no en vano, en solo tres cursos hemos pasado de 6 a 47 centros implicados, y de 1 sector a 8 sectores. La perspectiva es de seguir creciendo y avanzando a $6^{\circ}$ curso y a otros sectores nuevos, especialmente porque lo que nos mueve es compartirlo y que más personas puedan verse beneficiadas.

Comprobamos que el nivel de motivación y aprendizajes adquiridos, así como servicio prestado a la comunidad es valioso, y nos gustaría continuar consolidándolo y potenciándolo en cada centro, comunidad y sector. Es altamente satisfactorio experimentar el servicio a la comunidad en las valoraciones positivas de compañeros que desean 
llevar a cabo el proyecto en nuevos centros y sectores. Nos encantaría poder seguir compartiéndolo con más personas y comunidades. Por ello, confiamos en propiciar más vida activa, más salud, más actividad física, más amigos y más momentos sanos, divertidos, inclusivos, solidarios y de éxito para todo el alumnado, especialmente en su tiempo de ocio, fomentando en comunidad la vida activa en todos sus ámbitos.

\section{Referencias bibliográficas}

Aramburuzabala, P. (2013).

Aprendizaje-servicio: una herramienta para educar desde y para la justicia social. Revista internacional de educación para la justicia social, 2(2), 5-11.

Batlle, R. (2011). ¿De qué hablamos cuando hablamos de aprendizaje servicio? Crítica, 972, 49-54.

Campo, L. (2015). Evaluar para mejorar los proyectos de aprendizaje servicio en la universidad. RIDAS, Revista Iberoamericana de Aprendizaje y Servicio, 1, 91-111.

Cofiño, R.; Aviñó, D.; Benedé, C.B.; Botello, B.; Cubillo, J.; Morgan, A.; Paredes-Carbonell, J.J. y Hernán, M. (2016). Promoción de la salud basada en activos: ¿cómo trabajar con esta perspectiva en intervenciones locales? Gaceta sanitaria, 30(S1), 93-98.

Devís, J. y Peiró, C. (1993). La actividad física y la promoción de la salud en niños/as y jóvenes: la escuela y la educación física. Revista de psicología del deporte, 2(2), 71-86.
Dotterweich, A.R.; Green, A. y Blosser, D. (2012). Using innovate playgrounds and cross-curricular design to increase physical activity. Journal of Physical Education, Recreation \& Dance, 83(5), 47-55.

Frago, J.M. (2015). Niveles de actividad física en escolares de educación primaria: actividad física habitual, clases de educación física y recreos. Tesis doctoral. Universidad de Zaragoza.

García Ferrando, M. y Llopis, R. (2017). La popularización del deporte en España. Encuestas de hábitos deportivos (1980-2015). Madrid: Consejo Superior de Deportes y Centro de Investigaciones Sociológicas.

Global Advocacy for Physical Activity, GAPA (2010). La Carta de Toronto para la Actividad Física: Un llamado global para la Acción. Disponible en http://activate.gob. mx/documentos/car ta\%20de\%20toronto\%20para\%20la\%2 Oactividad\%20fisica.pdf

González-Cutre (2017). Estrategias didácticas y motivacionales en las clases de educación física desde la teoría de la autodeterminación. eMotion. Revista de Educación, Motricidad e Investigación, 8, 44-62.

Hattie, J. y Anderman, E.M. (2013). International guide to student achievement. New York: Routledge.

Hernández, L.A. (2009). Análisis de la actividad física de los escolares de primaria y secundaria de la ciudad de Zaragoza. Tesis doctoral. Universidad de Zaragoza. 
Julián, J.A. (2012). Motivación e intervención docente en la clase de educación física. Tándem, 40, 7-17.

Langille, J. L. D y Rodgers, W. M. (2010). Exploring the influence of a social ecological model on school-based physical activity. Health Education \& Behavior, 37(6), 879-894.

Navarro, A.; Martín, D.; García, C.; Jofre, E.; Hernández, P. y Gómez, D. (2017). Proyecto amigos activos. Una propuesta de actividad física inclusiva. Tándem, 55, 59-65.

Martín, D.; Navarro, A.; Bona, D.; Félix, M.; Gutiérrez, V.; Pérez, S.; Rodríguez, F.; Rodríguez, J.L.; Torres, G. y Vidal, S. (2018). Proyecto Amig@s Activ@s:

Aprendizaje Servicio y Acción comunitaria solidaria. Fórum Aragón, 25, 83-89.

Murillo, B.; García-Bengoechea, E.; Generelo, E.; Bush, P.L.; Zaragoza, J.; Julián, J.A. y García-González, L. (2013). Promising school-based strategies and intervention guidelines to increase physical activity of adolescents. Health Education Research, 28(3), 523-538.

Ojiambo, R.M. (2013). Physical Activity and Well-being: A review of the Health Benefits of Physical Activity on Health Outcomes. Journal of Applied Medical Sciences, 2(2), 69-78.

Orden de 16 de junio 2014, de la Consejera de Educación, Universidad, Cultura y Deporte, por la que se aprueba el currículo de la Educación Primaria y se autoriza su aplicación en los centros docentes de la Comunidad
Autónoma de Aragón. Publicado en B.O.A. no 119, de 20 de junio.

Organización Mundial de la Salud (1986). Carta de Ottawa para la promoción de la salud. Copenaghe: OMS. Recuperado de http://www.fmed.uba.ar/depto/toxico1/ carta.pdf

Organización Mundial de la Salud (2010): Recomendaciones mundiales sobre actividad física para la salud. Ginebra. OMS. Recuperado de http://apps.who.int/iris/ bitstream/10665/44441/1/9789243599 977 spa.pdf

Pérez Juste, R. (2006). Evaluación de programas educativos. Madrid: La Muralla.

Puig, J.M. ; Batlle, R. ; Bosch, C. y Palos, J. (2007). Aprendizaje servicio. Educar para la ciudadanía. Barcelona:

Octaedro.

Puig, J.M. ; Martín, X.; Rubio, L.; Palos, J.; Gijón, M.; De la Cerda, M. y Graell, M. (2014). Rúbrica para la autoevaluación y mejora de los proyectos ApS. Barcelona: Fundación Jaume Bofill. Recuperado de http://www.aprenentatgeservei.org/intr a/aps/documents/ aps autoevaluacio cast IMP A5.pdf

Puig, J.M. (coord) (2015). 11 ideas clave. ¿Cómo realizar un proyecto de aprendizaje servicio? Barcelona: Graó.

Ramírez, W.; Vinaccia, S. y Suárez, G. R. (2004). El impacto de la actividad física y el deporte sobre la salud, la cognición, la socialización y el 
rendimiento académico: una revisión teórica. Revista de Estudios Sociales, (18), 67-75.

Rojas, M. (2013). Convergencia:

Educación para el desarrollo desde la comunicación social. Zaragoza:

Fundación Familias Unidas. Recuperado

de

https://issuu.com/sensibilizacioncondos orillas/docs/convergencia b

Sallis, J.F.; Owen, F. y Fisher, E.B. (2008). Ecological models of health behavior. En: K. Glanz, B. Rimer y K. Viswanath (Eds.). Health behavior and health education: theory, research, and practice (pp. 465-482). San Francisco: Jossey-Bass.

Solmon, M.A. (2015). Optimizing the Role of Physical Education in Promoting Physical Activity: A Social-Ecological Approach. Research Quarterly for Exercise and Sport, 86, 329-337.

UNESCO (2015). Replantear la educación. ¿Hacia un bien común mundial? París: UNESCO. Recuperado de

http://unesdoc.unesco.org/images/002 3/002326/232697s.pdf 